\title{
AN ARTIFICIAL NEURAL NETWORK MODEL FOR EFFICIENT ESTIMATION OF THE NUMBER OF MOBILE STOCHASTIC EM SOURCES IN THE SPACE SECTOR
}

\author{
Zoran Stanković, \\ Ivan Milovanović \\ Nebojša Dončov', \\ Bratislav Milovanović2
}

${ }^{1}$ University of Niš, Faculty of Electronic Engineering, Niš, Serbia

${ }^{2}$ Singidunum University, Belgrade, Serbia

Correspondence:

Ivan Milovanović

e-mail:

imilovanovic@singidunum.ac.rs

\begin{abstract}
:
Information on the total number of radiation sources that are currently observed in the physical sector may be of use in procedures dealing with efficient DoA (Direction of Arrival) estimation of stochastic radiation source. This paper introduces an artificial neural model based on MLP (Multi-Layer Perceptron) network, that is based on a value of the spatial correlation matrix signal sampled in the far zone ofradiation, and can determine in real time, the number of mobile stochastic sources (up to five sources) with mutually uncorrelated radiation that are currently present in the given sector in the azimuthal plane. This model is an upgrade MLP model that previously had implementation for a maximum of three sources in the sector. Training neural model was carried out on the samples of the spatial correlation matrix obtained by using Green's functions. The authors observe the case when the number of sources in the sector variable in time and when the sources during the movement in the given sector may be located in another arbitrary distance.
\end{abstract}

Key words:

multilayer perceptron (MLP), antenna system,

artificial neural network, wireless communication system.

\section{INTRODUCTION}

One of important activities that open the possibility of further increasing the number of users of modern wireless communication and quality of service which is offered to customers of such systems are, researching methods to effectively minimize the negative impact of interference on the site of received signals. In this sense, the procedures based on spatial filtering, signal antenna arrays and design characteristics of radiation using adaptive antenna arrays now receive special attention $[1,2]$. The above-mentioned processes show some of the key techniques that implement such procedures based on DoA estimation techniques [1.2], and the spatial localization of various sources of interference as deterministic and stochastic nature of radiation $[3,4]$.

MUSIC (Multiple Signal User Classification) algorithm [2], and its modifications are so far the best known and most commonly used superresolution algorithms for DOA estimation. These algorithms are characterized by a high degree of accuracy in determining the direction where EM signals come from. However, because of its complex matrix calculation requires powerful hardware resources, they are not suitable for 
real-time operations. A good alternative to the superresolution algorithms, the application of artificial neural networks [5-7] to solve problems DoA where neural models that avoid complex matrix calculations can have an approximate accuracy of the MUSIC algorithm without having to be significantly faster than the MUSIC algorithm which makes them more suitable choice for deployment in real time [8-17].

In the process of DoA estimation, using either superresolution algorithm or neural model, the knowledge of the number of sources of EM signals (radiation) represents important information that helps in the implementation of an efficient and accurate process. In the works $[16,17]$ developed a neural model based on PNN (Probabilistic Neural Network) [18-20] that on the basis of the value of spatial correlation matrix signal which is sampled in the far zone of radiation, can determine in real time the number of mobile stochastic sources with mutually uncorrelated radiation that are currently present in the given sector in the azimuthal plane. The performance of our PNN model is shown in the examples were satisfactory, but it was concluded that increasing the maximum number of sources that can be found in the observed sector significantly complicates the structure of PNN model, which can lead to significant performance degradation neuronal models in terms of training and achieve satisfactory accuracy in the process of exploitation of models. This is primarily because the increase in the maximum number of sources that can be found in the observed sector dramatically increases the number of samples for training the neural model, and therefore, the complexity of used PNN where the number of neurons in the hidden layer in direct proportion to the number of samples for training models.

Bearing in mind the above mentioned limitations, the PNN model began with examining the possibilities of applying MLP network $[5,6]$ as one of the alternative PNN in the process of determining the number of mobile stochastic sources with mutually uncorrelated radiation that are currently present in the given sector in the azimuthal plane. When selecting alternatives, it is necessary to take into account the fact that with an increasing number of samples, for training an MLP network, complexity grows to a much lower degree, compared to the growth of complexity of a PNN, because the number of neurons in the hidden layer of an MLP network is not in direct proportion to the number of samples, and in a number of samples an MLP network can have manifold smaller number of neurons in the hidden layer in relation to a PNN. The first results were achieved in [21], where the MLP neural model to determine the number of the source under the condition that a maximum of three sources can be found at the same time in the sector. This paper presents a MLP model trained to determine the number of sources in the sector under the condition that a maximum of five sources can simultaneously occur in the given sector and which was created to further the development and training of the MLP model, which was introduced in [21].

\section{MODEL RADIATION SOURCES IN STOCHASTIC FAR ZONE}

To generate a set for training a neural model, and a set of samples to test it, the same model of stochastic radiation source in the remote zone is applied, as in the papers [10-17]. The model of stochastic radiation sources in the distant zone represents radiation of a uniform linear antenna array of $\mathrm{N}$ elements located at the distance $d$ (Figure 1). The degree of correlation between the current supply element antenna array that is described by the vector $\left.\mathrm{I}=\left[I_{1}, I_{2}, \ldots, I_{N}\right]\right)$, is defined by the correlation matrix $c^{I}(\omega)[3,4]$ :

$$
\mathbf{c}^{I}(\omega)=\lim _{T \rightarrow \infty} \frac{1}{2 T}\left[I(\omega) I(\omega)^{H}\right]
$$

In the zone of far-field electric field, the strength at the selected point is calculated in the following way:

$$
E(\theta, \varphi)=\mathbf{M}(\theta, \varphi) \mathbf{I}
$$

where $\mathbf{M}$ represents the mapping Green's function:

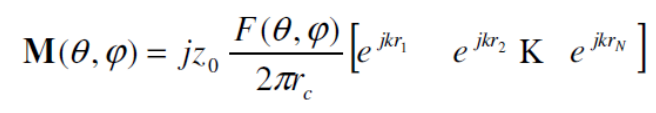

In the previous equations, $\theta \& \varphi$ represent the corners of the selected point in the azimuthal and elevational plane compared to the first antenna element, $F(\theta, \varphi)$ is the radiation characteristics of the antenna element, $\mathrm{rc}$ is the distance between the selected points of the array center, $z_{0}$ is the impedance of free space, $k$ is the phase constant $(k=2 \pi / \lambda)$, while $r_{1}, r_{2}, \ldots, r_{N}$ represent the distance to the selected point from the first to the $\mathrm{N}$-th antenna element respectively.

In the case that in the distant zone we sample values of the electric field intensity at more points, we use even more complex notation to indicate the distance. For example, in Figure 1. $r_{i, m}$ the distance between the i-th array element $(1 \leq i \leq N)$ and $m$-th sampling points in a distant zone $(1 \leq m \leq M)$, where $\mathrm{M}$ is the number of sampling points. 


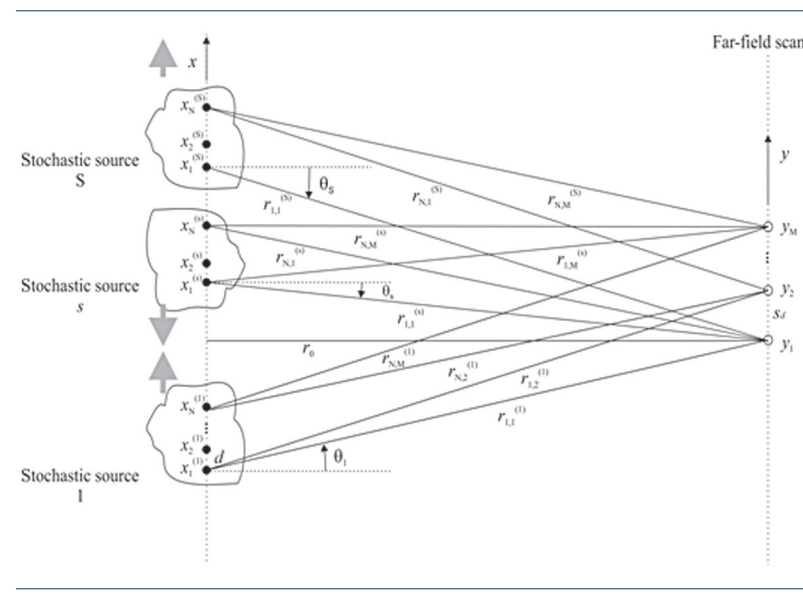

Figure 1. Model of stochastic radiation source in a distant zone showing the appropriate distance between sampling points and elements of antenna arrays that represent sources of radiation [10-17]

If the corresponding angles in the azimuthal and elevation level of $M$ sampling points $\left(y_{1} y_{2}, \ldots, y_{M}\right)$ in far zone represent with $\left(\theta_{1^{\prime}} \varphi_{1}\right),\left(\theta_{1} \varphi_{1}\right), \ldots,\left(\theta_{M^{\prime}} \varphi_{M}\right)$ respectivly, then the correlation matrix of signals at these points can be determined in the following manner: [8]

$$
\tilde{\mathbf{C}}_{E}[i, j]=\mathbf{M}\left(\theta_{i}, \varphi_{i}\right) \mathbf{c}^{I} \mathbf{M}\left(\theta_{j}, \varphi_{j}\right)^{H}, i=1, \ldots, M \quad j=1, \ldots, M
$$

In the case of multiple sources where $S$ is the number of sources, matrix $\mathbf{M}$ has the following layout:

$$
\mathbf{M}(\theta, \varphi)=j z_{0} \frac{F(\theta, \varphi)}{2 \pi_{c}} \cdot\left[e^{j k_{1}^{(1)}} \mathrm{K} e^{j k_{N}^{(1)}} e^{j k_{1}^{(2)}} \mathrm{K} e^{j k_{N}^{(2)}} \mathrm{K} e^{j k k_{1}^{(s)}} \mathrm{K} e^{j k_{N}^{(s)}}\right]
$$

where $r_{i}^{(j)}$ is the distance between the $\mathrm{i}$-th element antenna array that represents the $j$-th generation source and the observed point sampling in a distant zone so that the vector power supply is as follows:

$$
\mathbf{I}=\left[I_{1}^{(1)} \mathrm{K} I_{N}^{(1)} I_{1}^{(2)} \mathrm{K} I_{N}^{(2)} \mathrm{K} I_{1}^{(S)} \mathrm{K} I_{N}^{(S)}\right]
$$

where $I_{i}^{(j)}$ represents the power supply of the $i$-th element antenna array that represents the $j$-th generation source. The elements of the correlation matrix that is related to the sampling points $\widetilde{\mathbf{C}}_{E}$ can be calculated when the equations (1) and (5) used for calculating the correlation matrix using equation (4).

If two sources of radiation have the same angles $(\theta, \varphi)$ but are located at different distances from the observed point in the distant zone $r_{c 1}$ and $r_{c 2}$ respectively, and if the sources are represented as antenna arrays with $N_{1}$ and $N_{2}$ elements respectively, then it can be shown that their correlation matrix is valid

$$
\tilde{\mathbf{C}}_{E 2} \approx \frac{N_{2} r_{c 1}}{N_{1} r_{c 2}} \cdot \tilde{\mathbf{C}}_{E 1}
$$

for the fulfillment of the conditions $r_{c 1}, r_{c 2}>d$. Normalization of the matrix $\widetilde{\mathbf{C}}_{E}$, with respect to the first element of the matrix $\widetilde{C}_{E 11}$, the matrix is obtained where its elements do not depend on the value of the $r_{c}$ and $N[16,17]$. For training the neural network which the model contains, it is enough to take only the first level of matrix $C_{E}\left(\left[C_{E 11}, C_{E 12}, \ldots, C E_{I M}\right]\right)$ because it turned out that the first type contains sufficient information to determine the angular position of the radiation source $[9,10]$.

\section{ARCHITECTURE OF MLP NEURAL MODEL TO DETERMINE THE NUMBER OF SOURCES OF RADIATION}

According to the theory presented in the previous section, the problem of the modeled neural model will be presented in the following functional form:

$$
s=f\left(\mathbf{C}_{E}\right)
$$

Accordingly, the artificial neural network has a task to perform mapping from space signal which describes the correlation signal matrix $\mathrm{CE}$, the discrete space containing the numbers of radiation sources that can be found in the observed sector in the azimuthal level where variable indicates the number of sources that are currently in the sector and where $s \in\{1,2, \ldots, S\}$ and $S$ represents the maximum number of sources of radiation, which at one point can be found in the given sector.

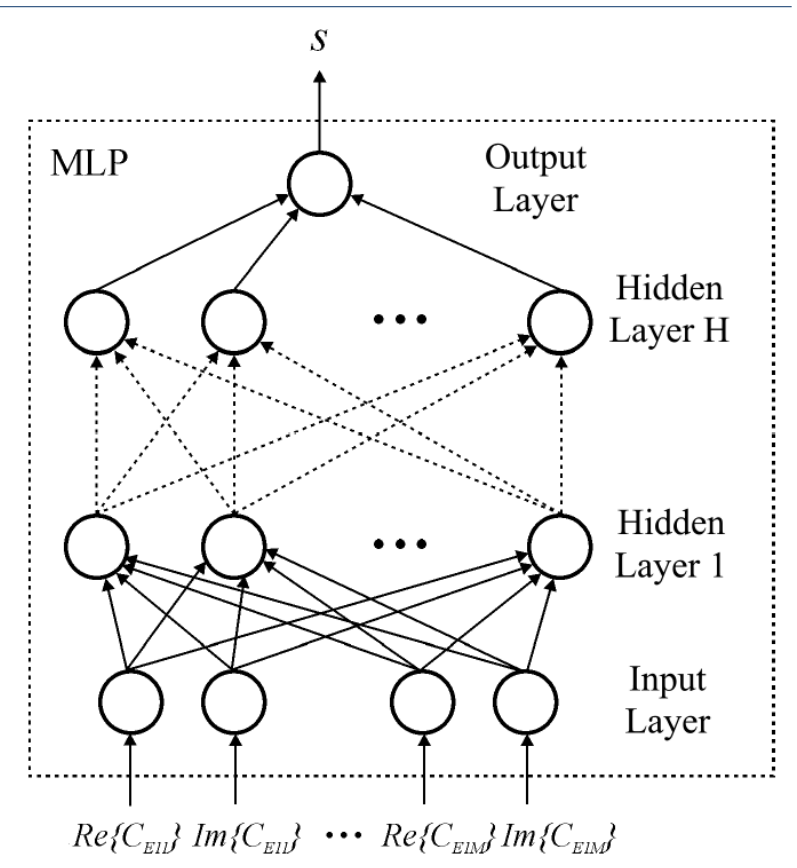

Figure 2. Architecture MLP neural model for determining the number of stochastic radiation sources in the reporting sector in the azimuthal level [21] 
As already stated in the introductory sector, it is not necessary to use the value of all elements of the correlation matrix signal for satisfactory approximation of the mappings, but is possible to use only the first type $C_{E}[1, i], i=1 \ldots M[8]$. Consequently, the number of inputs to the neural network will be $2 M$, because entries in the neural network cannot be complex numbers, but only real. Furthermore, the neural model that is functionally described as $\mathbf{y}=y(\mathbf{x}, \mathbf{w})$, where $\mathrm{y}$ is it proper function of neural networks and the weight matrix w neural networks [5,6], will have a vector of inputs $x=\left[R_{e}\left\{c_{E 1}\right\}\right.$, $\left.I_{m}\{c E 11\}, R_{e}\left\{c_{E 12}\right\}, I_{m}\left\{c_{E 12}\right\} \ldots R_{e}\left\{c_{E I M}\right\}, \operatorname{Im}\left\{c_{E I M}\right\}\right]^{\mathrm{T}}$, while the output value vector is $\mathbf{y}=s$. Since the implementation models used MLP network that has a continuous output of real value, the corresponding MLP neural model will be defined in the following way:

$$
\begin{aligned}
s & =\operatorname{round}\left(y\left(\left[\operatorname{Re}\left\{c_{E 11}\right\}, \operatorname{Im}\left\{c_{E 11}\right\}, \mathrm{K}, \operatorname{Re}\left\{c_{E I M}\right\}, \operatorname{Im}\left\{c_{E I M}\right\}\right]^{T}, \mathbf{w}\right)\right. \\
& \left.=f_{M L P}\left(\operatorname{Re}\left\{c_{E 11}\right\}, \operatorname{Im}\left\{c_{E 11}\right\}, \mathrm{K}, \operatorname{Re}\left\{c_{E 1 M}\right\}, \operatorname{Im}\left\{c_{E I M}\right\}, W\right)\right)
\end{aligned}
$$

where fMLP transfer function or function processing MLP network while round function rounds out the real value of output MLP network to discrete integer value representing the number of radiation sources with $s$.

The architecture of the MLP neural model to determine the number of stochastic radiation sources in the monitored sector in the azimuthal plane is shown in Figure 2. Exit of the $h$-th hidden layer MLP network by which this model is realized, can be represented by vector $\mathbf{y}_{h}$ with dimmensions $N_{h} \times 1$ where is $N_{h}$ the number of neurons in $h$-th hidden layer, and where the $i$-th element in that vector $-\mathbf{y}_{h}[i]$ represents the exit of $i$-th neuron $l$-th layer of the network $(l=h+1$ and counting the input layer) $v_{i}^{(l)}=v_{i}^{(h+1)}$, also valid $\mathbf{y}_{l}=\left[v_{1}^{(h+1)}, v_{2}^{(h+1)}, \mathrm{K}, v_{N_{l}}^{(h+1)}\right]^{\mathrm{T}}$. It can be shown that the vector is

$$
\mathbf{y}_{h}=F\left(\mathbf{w}_{h} \mathbf{y}_{h-1}+\mathbf{b}_{h}\right)
$$

where $y_{l-1}$ is the vector of dimension $N_{l-1} \times 1$ and represents the exit of the (h-1)-th hidden layer, wh the weight matrix of connections between neurons $(h-1)$-th and $h$-th hidden layer dimensions $N_{h} \times N_{h-1}$, where as $\mathbf{b}_{h}$, is the vector of the bias $h$-th hidden layer. According to this notation, $\mathbf{y}_{0}$ represents the output of the input buffer layer so that $\mathbf{y}_{0}=x$. Element $\mathbf{w}_{h}[i, j]$ of the weight matrix $\mathbf{w}_{l}$ marks the weight of the link between the $h$-th neuron in the hidden layer $(h-1)$ and $h$-th neuron in the hidden layer $h$, i.e. between $h$-th neuron in the network layer $s=h$ and $j$-th neuron in the network layer $l=h+1$, while element $b_{i}^{(h)}=\mathbf{b}[i]$ represents the value of the bias of the $i$-th neuron in the hidden layer $h$. The function $F$ represents the activation function of neurons in the hidden layers. In this case, tangent hyperbolic sigmoid function is used.

$$
F(u)=\frac{e^{u}-e^{-u}}{e^{u}+e^{-u}}
$$

All neurons in the last hidden layer are connected to the $H$ output layer neuron. The activation function of the neurons in the last layer is linear and the output MLP network is:

$$
y=\mathbf{w}_{o} \mathbf{y}_{H}
$$

where wo weight matrix connects between neurons of the $H$-th hidden layer, and neurons from the exit layer, measuring $1 \times N_{H}$ (Figure 2). The final output of the neural model produced by rounding output MLP neural network to the nearest whole number:

$$
s=\operatorname{round}(y)
$$

Indication of such defined MLP neural model that will continue to be used in the text is $M L P H-N_{1}-\ldots-N i-\ldots-N_{H}$ where $H$ is the total number of hidden layers used in the MLP network, while Ni is the total number of neurons in the i-th hidden layer. Thus, tag MLP2-14-12 marks a MLP model indicates which neural network has four neural layers (input, output and two hidden layers) and which has 14 neurons in the first hidden layer and 12 neurons in the second hidden layer.

\section{MODELING RESULTS}

MLP neural model whose architecture was shown in the previous section has been applied to determine the number of stochastic radiation sources in the azimuthal sector $\left[-30^{\circ} 30^{\circ}\right]$. The case was studied where, up to five radiation sources can be found in the given sector, at one point of time. One stochastic source is modeled as an antenna array of four elements, but in order to determine the correlation matrix signal in the far zone, radiation is carried out in the six-point sampling at a distance $\mathrm{sd}=1 / 2=0.02 \mathrm{~m}$ at $\mathrm{f}=7.5 \mathrm{GHz}$ (Table 1$)$.

\begin{tabular}{ll}
\hline Frequency & $f=7.5 \mathrm{GHz}$ \\
\hline Max sources & $S=5$ \\
\hline $\begin{array}{l}\text { Number of elements in the antenna } \\
\text { array of one source }\end{array}$ & $N=4$ \\
$\begin{array}{l}\text { The distance between the elements of } \\
\text { the antenna array }\end{array}$ & $d=\lambda / 2(0.02 \mathrm{~m})$ \\
\hline $\begin{array}{l}\text { The distance from the source to the } \\
\text { stochastic sampling in the far zone }\end{array}$ & $r_{0}=1000 \mathrm{~m}$ \\
\hline $\begin{array}{l}\text { Number of sampling sites in the } \\
\text { distant zone }\end{array}$ & $M=8$ \\
\hline The distance between sampling points & $s_{d}=\lambda / 2(0.02 \mathrm{~m})$ \\
\hline
\end{tabular}

Table 1. Parameter values of antenna array which represent stochastic sources and methods of sampling in the far zone of radiation sources 
For the realization of the training model, MatLab 7.0 software development environment was used. The sets of samples for training and testing MLP models were generated using the inverse mapping from the one who does the MLP model

$$
\mathbf{C}_{E}^{t}=f_{D o A}^{-1}\left(\boldsymbol{\theta}^{t}\right)
$$

and which is realized by the equations (4) and (5). In the above mentioned mapping, $\boldsymbol{\theta}^{\mathrm{t}}$ represents the vector of angular position of the radiation source $\left[\theta_{1}^{t}, \theta_{2}^{t}, \ldots, \theta_{s}^{t}\right]$. Since the input of the neural network results in only the first type correlation matrix, training and test samples will take the form of $\left(\mathbf{x}^{t}\left(\boldsymbol{\theta}^{t}\right), s\right)$ where the vector inputs training and test sets applies

$$
\mathbf{x}^{t}\left(\boldsymbol{\theta}^{t}\right)=\left[\operatorname{Re}\left\{C_{E 11}^{t}\right\} \operatorname{Im}\left\{C_{E 11}^{t}\right\} \operatorname{Re}\left\{C_{E 12}^{t}\right\} \operatorname{Im}\left\{C_{E 12}^{t}\right\} \operatorname{K} \operatorname{Re}\left\{C_{E 1 M}^{t}\right\} \operatorname{Im}\left\{C_{E 1 M}^{t}\right\}\right\}
$$

A uniform distribution of samples for training and testing MLP model in the sector $\left[-30^{\circ} 30^{\circ}\right]$ was applied in a manner: $\left[-30^{0}: d: 30^{\circ}\right]$ where $d$ is a step of sampling, meetings for training and testing MLP model are described as follows[17]:

$\left\{\left(\mathbf{x}^{t}\left(\theta_{1}^{t}\right), 1\right) \mid \theta_{1}^{t} \in\left[-30: d_{1}: 30\right]\right\} \cup$

$\left\{\left(\mathbf{x}^{t}\left(\theta_{1}^{t}, \theta_{2}^{t}\right), 2\right) \mid \theta_{1}^{t} \in\left[-30: d_{2}: 30\right], \theta_{2}^{t} \in\left[-30: d_{2}: 30\right], \theta_{1}^{t}>\theta_{2}^{t}\right\} \cup$

$\left.\int\left(\mathbf{x}^{t}\left(\theta_{1}^{t}, \theta_{2}^{t}, \theta_{3}^{t}\right), 3\right) \mid \theta_{1}^{t} \in\left[-30: d_{3}: 30\right], \theta_{2}^{t} \in\left[-30: d_{3}: 30\right],\right\}$

$\left\{\theta_{3}^{t} \in\left[-30: d_{3}: 30\right], \theta_{1}^{t}>\theta_{2}^{t}>\theta_{3}^{t}\right.$

$\left.\int\left(\mathbf{x}^{t}\left(\theta_{1}^{t}, \theta_{2}^{t}, \theta_{3}^{t}, \theta_{4}^{t}\right), 4\right) \mid \theta_{1}^{t} \in\left[-30: d_{4}: 30\right], \theta_{2}^{t} \in\left[-30: d_{4}: 30\right],\right\}$

$\left\{\theta_{3}^{t} \in\left[-30: d_{4}: 30\right], \theta_{4}^{t} \in\left[-30: d_{4}: 30\right], \theta_{1}^{t}>\theta_{2}^{t}>\theta_{3}^{t}>\theta_{4}^{t}\right\} \cup$

$\int\left(\mathbf{x}^{t}\left(\theta_{1}^{t}, \theta_{2}^{t}, \theta_{3}^{t}, \theta_{4}^{t}, \theta_{5}^{t}\right), 5\right) \mid \theta_{1}^{t} \in\left[-30: d_{5}: 30\right], \theta_{2}^{t} \in\left[-30: d_{5}: 30\right]$,

$\left\{\theta_{3}^{t} \in\left[-30: d_{5}: 30\right], \theta_{4}^{t} \in\left[-30: d_{5}: 30\right], \theta_{5}^{t} \in\left[-30: d_{5}: 30\right]\right.$,

$\theta_{1}^{t}>\theta_{2}^{t}>\theta_{3}^{t}>\theta_{4}^{t}>\theta_{5}^{t}$

where generating the set for training takes, $d_{1}=0.1$, $d_{2}=0.5, d_{3}=2, d_{4}=4, d_{5}=6$, whereas, for generating the set for testing takes, $d_{1}=0.13, d_{2}=0.7, d_{3}=3, d_{4}=7, d_{5}=11$. With this distribution, the set for training is generated with, 14638 samples, and a test set of 5579 sample.

For the training model, Quazi-Newton method of training was used. In order to obtain the best possible MLP model, a training was conducted for a number of MLP neural networks, with two hidden layers and different numbers of neurons in them. In the process of testing the main criterion for evaluating the success of training, is the percentage value of samples for which determined a wrong number of present sources of radiation in the sector, or shorter, percentage value of samples that war misclassified (UPK [\%]) [16,17]. In order to compare the test results of the model, Table 2 presents the testing results of six MLP models, that are on the same set of tests, and had the lowest percentage of samples that are misclassified. In this table, the UTK in- dicates the number of samples for which the MLP model gave the exact number of present sources of radiation in the sector and with UPK indicating the number of samples for which the MLP model gave the wrong number of present sources of radiation in the sector.

\begin{tabular}{cccc}
\hline MLP model & UTK & UPK & UPK [\%] \\
\hline MLP2-22-20 & 5445 & 134 & 2.46 \\
\hline MLP4-22-20 & 5438 & 141 & 2.59 \\
\hline MLP4-23-23 & 5443 & 136 & 2.50 \\
\hline MLP4-23-23 & 5434 & 145 & 2.67 \\
\hline MLP4-22-20 & 5445 & 134 & 2.46 \\
\hline MLP4-22-20 & 5444 & 135 & 2.48 \\
\hline
\end{tabular}

Table 2. Testing resaults of six MLP models with the best resaults when it comes to determining the correct number of sources in the sector

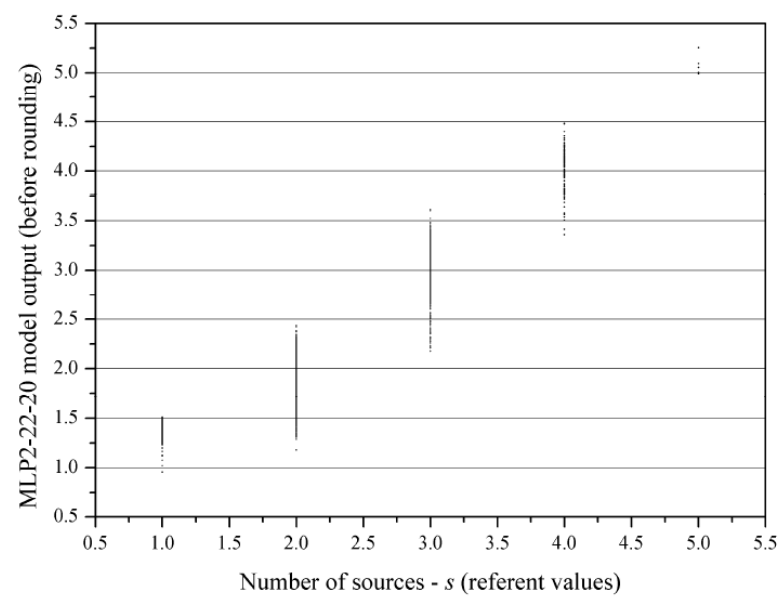

Figure 4. Diagram of dissipation MLP2-22-22 models on the test set when determining the number of present sources of radiation in $\left[-30^{\circ} 30^{\circ}\right]$ sector

As a representative model for determining the number of stochastic radiation sources in the given sector, in the azimuthal plane, the model of MLP2-22-22, was chosen. The model had the lowest percentage of misclassified samples, and that has shown the highest success rate in determining the number of radiation sources in the given sector. This model is shown in Figure 4.

It can be observed that the accuracy of the MLP model for the case of $S=5$, is as approximately accurate as the MLP models that have been developed for the case of $S=3$ [21]. This shows that the MLP model maintained good performances in modeling problems in spite of increasing complexity of the problem, and consequently, a significant increase in the number of samples for training. 


\section{CONCLUSION}

In this paper, the examination of the possibility of MLP neural networks was continued to determine the number of radiation sources in the given sector in the azimuthal level that began in [21], with an increase of the maximum number of sources that can be found in the sector, simultaniously, from three to five sources. Due to the avoidance of complex numerical calculation, it is also shown that MLP model can quickly and with high accuracy determine the number of radiation sources in the observed sector. An increase in the maximum number of radiation sources that are simultaneously found in the observed sector, inevitably leads to a significant increase in the number of samples which are necessary for training the neural model, that will be able to process such a number of sources (8921 samples for 3 sources 14638 samples of 5 sources), and the fact that, with an increasing number of samples complexity of the PNN is growing much faster, than the complexity of the MLP network which can lead to limitations in the application of the PNN for the large number of radiation sources. MLP networks are emerging as more suitable variant to the PNN with a tendency to further increase the maximum number of sources of radiation that can be simultaneously found in the given sector.

\section{REFERENCES}

[1] R. Schmidt, "Multiple emitter location and signal parameter estimation", IEEE Transactions on Antennas and Propagation, vol. 34, no. 3, pp. 276-28, 1986.

[2] B. Allen, M. Ghavami, Adaptive Array Systems: fundamentals and applications, Wiley, 2005

[3] J.A. Russer, T. Asenov and P. Russer, "Sampling of stochastic electromagnetic fields", IEEE MTT-S International Microwave Symposium Digest, Montreal, Canada, pp. 1-3, 2012.

[4] J.A. Russer, P. Russer, "Modeling of Noisy EM Field Propagation Using Correlation Information", IEEE Transactions on Microwave Theory and Techniques, Volume 63, Issue 1, pp.76-89, 2015.

[5] S. Haykin, Neural Networks, New York, IEEE, 1994.

[6] Q. J. Zhang, K. C. Gupta, Neural networks for RF and microwave design, Artech House, Boston, MA, 2000.

[7] C. G. Christodoulou, M. Georgiopoulos, Application of neural networks in electromagnetics, Artech House, December 2000.
[8] A. H. El Zooghby, C. G. Christodoulou, M. Georgiopoulos, "A neural network based smart antenna for multiple source tracking", IEEE Transactions on Antennas and Propagation, Vol. 48, no. 5, pp. 768 776, 2000.

[9] M. Agatonović, Z. Stanković, N. Dončov, L. Sit, B. Milovanović, T. Zwick, "Application of artificial neural networks for efficient high-resolution 2D DOA estimation", Radioengineering, Vol. 21, No. 4, pp. 1178-1186, 2012.

[10] Z. Stanković, N. Dončov, J. Russer, T. Asenov and B. Milovanović, "Efficient DOA estimation of impinging stochastic EM signal using neural networks", Proceedings of the International Conference on Electromagnetics in Advanced Applications (15th Edition including EMS), ICEAA 2013, Torino, Italy, September 9-13, 2013, pp. 575-578, 2013.

[11] Z. Stanković, N. Dončov, J. Russer, I. Milovanović, M. Agatonović, "Neural networks based DOA estimation of multiple stochastic narrow-band EM sources", Proceedings of the 11th IEEE International Conference on Telecommunications in Modern Satellite, Cable and Broadcasting Services, TELSIKS 2013, Niš, Serbia 16-19 October, Vol. 2, pp. 526-529, 2013.

[12] Z. Stanković, N. Dončov, J. Russer, I. Milovanović, M. Agatonović, "Localization of Stochastic Electromagnetic Sources by using Correlation Matrix Trained MLP Neural Network", Microwave Review - National journal published by Society for Microwave Theory, Technology and Systems and IEEE MTT-S Chapter of Serbia and Montenegro, No.2, Vol. 19, pp. 44-49, December 2013.

[13] Z. Stanković, N. Dončov, J. Russer, I. Milovanović, B. Milovanović, "Neural Network Approach for Efficient DOA Determination of Multiple Stochastic EM Sources in Far-field", Proceedings of the 1st IEEE International Conference on Numerical Electromagnetic Modeling and Optimization for RF, Microwave, and Terahertz Applications, NEMO 2014, Pavia, Italy 14-16 May, 2014, pp.1-4, 2014.

[14] Z. Stankovic, N. Doncov, I. Milovanovic, B. Milovanovic, M. Stoiljkovic, "Localization of mobile users of stochastic radiation nature by using Neural Networks", Proceedings of the 49th International Scientific Conference on Information, Communication and Energy Systems and Technologies, ICEST 2014, Niš, Serbia, June 25 - 27, 2014, Vol. 2, pp. 347350, 2014.

[15] Z. Stankovic, N. Doncov, I. Milovanović, B. Milovanović, "Neural network model for efficient localization of a number of mutually arbitrary positioned stochastic EM sources in far-field", Proceedings of the 12th Symposium on Neural Network Applications in Electrical Engineering, NEUREL 2014, Beograd, Serbia, pp. 41-44, 2014. 
[16] Z. Stanković, N. Dončov, I. Milovanović, "Estimation of the Number of Stochastic EM Sources in Far-Field Using Probabilistic Neural Network", Proceedings of the 12th IEEE International Conference on Telecommunications in Modern Satellite, Cable and Broadcasting Services, TELSIKS 2015, Niš, Serbia 14-17 October, pp.377-380, 2015.

[17] Z. Stanković, N. Doncov, B. Milovanovic, I. Milovanovic, "Efficient DoA Tracking of Variable Number of Moving Stochastic EM Sources in FarField Using PNN-MLP Model," International Journal of Antennas and Propagation, vol. 2015, Article ID 542614, 11 pages, 2015. doi:10.1155/2015/542614

[18] D. F. Specht, "Probabilistic neural networks", Neural Networks, vol. 3, no. 1, pp. 109-118, 1990.
[19] K. Z. Mao, K.-C. Tan, and W. Ser, "Probabilistic Neural-Network Structure Determination for Pattern Classification", IEEE Transactions on Neural Networks, vol. 11, no. 4, pp. 1009-1016, july 2000.

[20] Ibrahiem M.M. El Emary and S. Ramakrishnan, "On the Application of Various Probabilistic Neural Networks in Solving Different Pattern Classification Problems", World Applied Sciences Journal 4 (6), IDOSI Publications, pp. 772-780, 2008.

[21] Z. Stanković, N. Dončov, I. Milovanovic, B. Milovanovic, "Estimation of the Number of Mobile Stochastic EM Sources in Space Sector using MultiLayer Perceptron Networks", Proceedings of the YUINFO 2016 Conference, Kopaonik, Serbia, February 28 -March 2, 2016. 